\title{
Spontaneous prospective-memory processing: Unexpected fluency experiences trigger erroneous intention executions
}

\author{
Jan Rummel $^{1}$ - Thorsten Meiser ${ }^{2}$
}

Published online: 25 August 2015

(C) Psychonomic Society, Inc. 2015

\begin{abstract}
Event-based prospective memory is the ability to remember to execute an intention when an environmental cue occurs. It has been argued that, due to their special meaning, these cues are discrepant from their environment and therefore are sometimes spontaneously noticed. In line with this assumption, the likelihood that an intention will be executed increases with increased cue-discrepancy. It is not yet clear, however, whether these improvements are due to facilitated spontaneous noticing rather than to an increase in the efficiency of controlled cue-processing. To further investigate the spontaneous nature of cue-discrepancy benefits, we presented participants with stimuli that were unrelated to the intention but discrepant from other stimuli. Therefore, we experimentally increased the processing fluency of some stimuli for participants currently holding an intention by using different priming procedures. We found that stimuli whose fluency was increased via spaced repeated stimulus presentation (Experiment 1) or via short pre-exposure (Experiment 2a to 3) elicited a tendency to fulfill the intention despite its actual inappropriateness. Findings were inconsistent as to whether cue-memory uncertainty fosters the reliance on cue discrepancy for intention retrieval (Experiments $2 \mathrm{a}$ and 3). Taken together, the present findings provide converging evidence for a spontaneous discrepancy-based prospective-memory process which works independent of controlled processes.
\end{abstract}

Jan Rummel

jan.rummel@psychologie.uni-heidelberg.de

1 Department of Psychology, Heidelberg University, Hauptstrasse 47-51, D-69117 Heidelberg, Germany

2 Department of Psychology, School of Social Sciences, University of Mannheim, Mannheim, Germany
Keywords Prospective memory $\cdot$ Spontaneous noticing · Processing fluency $\cdot$ Discrepancy-plus-search

The ability to remember to fulfill intentions at the appropriate moment in the future has been studied under the term prospective memory (PM; see McDaniel \& Einstein, 2007, for an overview). A typical example for a PM intention is remembering to buy groceries at the supermarket on the way home. In this example, the PM intention is associated with the occurrence of a certain event. Such event-based PM tasks require that an intention-related cue (e.g., the sign of the supermarket) is noticed and identified as a signal to execute the intention (cf. Graf, 2005). Event-based PM tasks are especially challenging for individuals who are engaged in other ongoing tasks (e.g., paying attention to the traffic) upon cue occurrence. Thus, the question arises how individuals manage to notice PM cues while performing ongoing tasks.

According to a multi-process view of PM (McDaniel \& Einstein, 2000, 2007), cue noticing can rely on two types of processes. One is controlled and attention demanding, the other is spontaneous and requires only minimal cognitive resources. For the former, attention must be devoted to monitoring the environment for cues. As attentional resources are limited (Cowan et al., 2005; Navon \& Gopher, 1979) such cue-monitoring should reduce the cognitive resources available for other tasks. In order to study PM processes in the laboratory, participants are typically asked to form the intention to respond with a specific key to certain cue stimuli which will occur in the context of a later to-be-performed ongoing task (Einstein \& McDaniel, 1990). Usually, ongoing-task performances of participants receiving an additional PM task are hampered compared to participants performing the ongoing task alone (Marsh, Hicks, Cook, Hansen, \& Pallos, 2003; Smith, 2003). These PM-induced costs indicate a reliance on 
controlled cue-monitoring. However, there is also evidence that intentions can re-enter consciousness spontaneously, that is, in the absence of cue-monitoring. For example, PM cues that are salient and/or fall naturally in the focus of attention are noticed and responded to, even when there are no ongoingtask costs observable (Einstein et al., 2005; Harrison \& Einstein, 2010; Rummel \& Meiser, 2013). The exact nature of the cognitive mechanism(s) allowing for spontaneous cuenoticing, however, is still an issue of debate.

In the present research, we seek new empirical evidence for a spontaneous PM process based on cue-discrepancy. This mechanism, which was first introduced by McDaniel and colleagues (McDaniel, Guynn, Einstein, \& Breneiser, 2004), builds on the discrepancy-attribution hypothesis by Whittlesea and Williams (2001a, 2001b). Whittlesea and Williams argue that individuals permanently appraise the (dis-)fluency of their processing and that this appraisal will produce a feeling of discrepancy if processing fluency differs from expectancies. Fluency is defined as the experienced ease with which a stimulus is perceptually processed (e.g., Johnston, Dark, \& Jacoby, 1985) and Whittlesea and Williams (2001a) assume that higherorder cognition can make use of this perceptual experience. Similarly, McDaniel et al. (2004) suggest that the processing of PM cues will feel discrepant from the processing of other environmental stimuli because the cues were encoded as being intention-relevant beforehand. This discrepancy experience will, in turn, trigger a memory search for possible causes, thereby bringing the intention back into mind. Empirical support for this discrepancy-plus-search process comes from findings that pre-exposing participants to future PM cues resulted in better PM performance compared to participants not being preexposed to the cues (Guynn \& McDaniel, 2007). Further, preexposure to all non-cue ongoing-task stimuli (but not to the cues) resulted in similar PM improvements, indicating that PM benefits are caused by the discrepancy between cues and their environment rather than by cue-familiarity per se (Breneiser \& McDaniel, 2006). Recently, Lee and McDaniel (2013) showed PM improvements from increased discrepancy between cue and non-cue stimuli which were due to mere processing-difficulty differences experienced on-the-fly while performing the ongoing task. Similarly, identity priming of PM cues (i.e., presenting a stimulus identical to the cue for a very short period just before the cue itself is presented) also increased PM performance (Graf, 2005). All these findings demonstrate PM benefits from increased discrepancy between cues and their environment (i.e., non-cue stimuli of an ongoing task). However, it is not clear whether cue-discrepancy benefits indeed indicate a spontaneous process. Alternatively, these benefits could be due to more efficient controlled processing, inasmuch as discrepancy facilitates the detection of the cues while being engaged in attentional cue-monitoring (see McDaniel \& Einstein, 2007, for a similar concern).
To further investigate the nature of discrepancy-based PM processing, we conducted a series of four experiments in which controlled and spontaneous processes were posed in opposition. For this purpose, we asked participants to form the intention to respond to certain cues with a specific action while performing an ongoing task. In addition, we made some non-cue stimuli discrepant from the other ongoing-task stimuli by increasing their processing fluency. This manipulation was inspired by the original discrepancy-attribution hypothesis assuming that higher-order cognition makes use of discrepancies between actual and expected processing fluency (Whittlesea \& Williams, 2001a). Assuming that PM also relies on fluency experiences to some extent, we hypothesized that fluent non-cue stimuli have the potential to reactivate intentions, because their processing feels discrepant from neutral (i.e., not discrepant) ongoing-task stimuli. Assuming that this fluency-based intention reactivation is a spontaneous process, we further hypothesize that unexpected fluency experiences will sometimes be (mis-)attributed as a signal to execute the intention, even though intention execution is not appropriate in this very moment. Importantly, a false intention execution caused by fluency variations of stimuli that have never been associated with the PM intention cannot be due to controlled cue-monitoring, because any controlled processing should prevent intention execution under such circumstances. In previous research using a similar rationale, false PM responses to cues that occur in a context where the intention is not relevant (Meiser \& Rummel, 2012) or after the intention has been canceled (Bugg \& Scullin, 2013; Scullin, Bugg, \& McDaniel, 2012) have been interpreted as indicators of a spontaneous process. In the present research, we go one step further and hypothesize that a fluently processed stimulus that has never been associated with an intention can spontaneously reactivate unfulfilled intentions, just because its processing differs from expectancies.

More specifically, in line with the conceptualization of a discrepancy-plus-search PM mechanism (McDaniel et al., 2004, see above), we assume that the fluent non-cues are noticed as discrepant and then trigger a (controlled) memory search for the source of this experience. Usually, in the course of this memory search, individuals will become aware that the fluent stimuli were not cues for intention execution and, consequently, the intended action will be withheld. However, in accordance with the general view that automatic processes prevail under suboptimal processing conditions whereas controlled processes do not (cf. Moors \& De Houwer, 2006a, 2006b), there may be situations in which a fluency-triggered intention execution cannot be prevented by subsequent controlled processing. According to Moors and De Houwer (2006b), automatic processes are not under conscious supervision and can thus be triggered by subliminal stimulus inputs. Further, unlike controlled processes, they still operate under time pressure or when cognitive resources are scarce. 
Building on this conceptualization of automaticity, we argue that false intention execution in response to unexpectedly fluent stimuli will be rare overall, but more likely under conditions that are "suboptimal" for a controlled memory search for the source of the fluency experience. To achieve such conditions, we used subtle manipulations of processing fluency to elicit a false (and thus spontaneous) intention execution and embedded the fluent stimuli in an ongoing continuousresponse task that required quick responding. Furthermore, we created conditions which should be suboptimal for a subsequent controlled processing (i.e., a controlled memory search for the true source of fluency after a fluency experience). For this purpose, we varied the subtlety of the source of fluency (cf. Jacoby \& Whitehouse, 1989). Further, inspired by findings that fluency effects on recognition memory are especially strong under memory uncertainty (Johnston, Hawley, \& Elliott, 1991), the number of cues associated with the intention was manipulated to test whether cue-memory uncertainty would foster the reliance on automatic PM processing.

\section{Overview of the present research}

Four experiments were conducted to test whether an unexpected processing-fluency experience has the potential to spontaneously reactivate unfulfilled intentions and to trigger a false intention execution. In all experiments, we realized a typical PM-task setting (cf. Einstein \& McDaniel, 1990) comprising four distinct phases: (1) formation of a PM intention, (2) a filler task delaying the intention, (3) an ongoing task with embedded PM cues, and (4) a retrospective intention-memory test (see Fig. 1). In all experiments, some non-cue ongoingtask stimuli were made discrepant from the remainder of the ongoing-task stimuli by increasing the fluency of their processing (i.e., PM lures). In Experiment 1, fluency of the lures was increased via repetition priming (e.g., Jacoby \& Whitehouse, 1989; Lloyd, Westerman, \& Miller, 2003). In order to vary the degree to which situations were suboptimal for a controlled search for the source of fluency and thus are susceptible to a misattribution of fluency (see above), the spacing between primes and lures was manipulated. In doing so, we aimed to test whether false intention execution becomes more likely with increased uncertainty about the origin of the lures' fluency (see Jacoby \& Whitehouse, 1989, for a simular argument regarding the reliance on fluency for recognition memory judgments). In Experiments 2a, 2b, and 3, more subtle identity priming was used to investigate whether fluent lures would trigger a false intention execution, even when they had not been presented before. Again, we aimed to create situations which were suboptimal for a controlled memory search after a fluency experience. For this purpose, in Experiments $2 \mathrm{a}$ and 3 the number of PM cues was manipulated to test whether the reliance on processing fluency would increase with increased cue-memory uncertainty (cf. Johnston et al., 1991). In Experiment 2b, the number of lures was increased, to further test the reliability of the lureinduced effect on false intention execution and to evaluate the assumed fast nature of the processing of the fluent lures (cf. Moors \& De Houwer, 2006b). In Experiment 3, challenges of the ongoing task were increased to replicate previous findings with different materials and to test whether lure-induced false intention execution would also occur when the ongoing task imposes additional workingmemory demands on the individual. Details of all four experiments are summarized in Table 1. For all experiments, we assured that the statistical power to detect medium-sized fluency effects was sufficiently high (i.e., $1-\beta>.80$; J. Cohen, 1968, assuming a correlation of .50 between repeated measures), which required sample sizes of at least $n=34$ in all experimental groups.

General time-course of Experiments 1-3

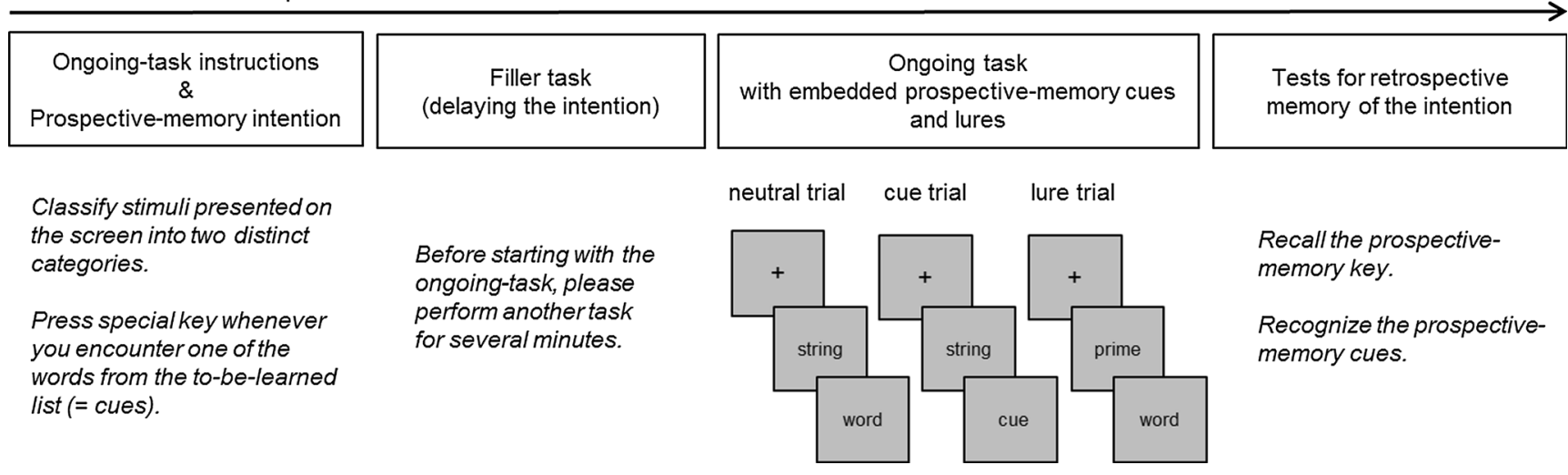

Fig. 1 Illustration of the general task setting and instructions (italicized) used in Experiments 1-4. The ongoing task was either a lexical-decision task (Experiments 1-2b) or a color-matching task (Experiment 3). Cues and cue-list length varied with experiments (see Table 1 for details). Lures were non-cue words whose processing fluency was increased via repetition priming (Experiment 1) or identity priming (Experiments 2a-3). Cue and lure trials occurred less frequently than neutral trials 
Table 1 Tasks and instructions used in Experiments 1-3

\begin{tabular}{|c|c|c|c|c|}
\hline & Experiment 1 & Experiment 2a & Experiment $2 \mathrm{~b}$ & Experiment 3 \\
\hline PM intention & $\begin{array}{l}\text { Press } 7 \text { when you see } \\
\text { a word from the list. }\end{array}$ & $\begin{array}{l}\text { Press U when you see } \\
\text { a word from the list. }\end{array}$ & $\begin{array}{l}\text { Press U when you see } \\
\text { a word from the list. }\end{array}$ & $\begin{array}{c}\text { Press } 1 \text { when you see a } \\
\text { word from the list. }\end{array}$ \\
\hline Cue-list length & 6 & 6 vs. 12 & 12 & 5 vs. 10 \\
\hline Filler-task & Raven matrices & Raven matrices & Raven matrices & Letter task \\
\hline Ongoing task & $\begin{array}{l}\text { Lexical-decisions: } \\
\text { Press A for words and } \\
\quad \text { L for nonwords }\end{array}$ & $\begin{array}{l}\text { Lexical-decisions: } \\
\text { Press A for words } \\
\quad \text { and L for nonwords }\end{array}$ & $\begin{array}{l}\text { Lexical-decisions: } \\
\text { Press A for words and } \\
\quad L \text { for nonwords }\end{array}$ & $\begin{array}{l}\text { Color-matching: } \\
\text { Press } J \text { if font color } \\
\text { matches preceding } \\
\text { squares and } N \\
\text { if not }\end{array}$ \\
\hline PM lures & $\begin{array}{l}\text { Stimuli being repeated } \\
\text { once with a lag of } \\
5 \text { vs. } 10 \text { trials }\end{array}$ & $\begin{array}{l}\text { Stimuli directly preceded } \\
\text { by an identity prime } \\
\text { of } 54 \mathrm{~ms}\end{array}$ & $\begin{array}{l}\text { Stimuli directly preceded } \\
\text { by an identity prime } \\
\text { of } 40 \mathrm{~ms}\end{array}$ & $\begin{array}{l}\text { Stimuli directly } \\
\text { preceded by } \\
\text { an identity prime } \\
\text { of } 42 \mathrm{~ms}\end{array}$ \\
\hline Number of lures & 4 & 6 & 33 & 10 \\
\hline $\begin{array}{l}\text { Intention-memory } \\
\text { test }\end{array}$ & $\begin{array}{l}\text { PM key recall and } \\
\text { cue recognition test }\end{array}$ & $\begin{array}{l}\text { PM key recall and cue } \\
\text { recognition test }\end{array}$ & $\begin{array}{l}\text { PM key recall and cue } \\
\text { recognition test }\end{array}$ & $\begin{array}{l}\text { PM key recall and cue } \\
\text { recognition test }\end{array}$ \\
\hline
\end{tabular}

Note. This is a general overview of the tasks used in the present research. Please refer to the corresponding Methods sections for more details.

\section{Experiment 1}

Experiment 1 was designed to test the hypothesis that an unexpected processing-fluency experience which is completely intention unrelated can spontaneously reactivate intentions and trigger a false intention execution. Therefore, a few noncue stimuli were presented repeatedly in the course of an ongoing task, while participants held a PM intention. If an unexpected fluency experience has the potential to reactivate intentions, participants should tend to respond with the intended action to the repeated stimuli. Additionally, we manipulated the number of intervening stimuli between the first and second presentation of the PM lures, to test whether the effect of processing fluency on false PM responses increases with a larger time lag between repeated presentations. In case of a larger time lag, participants are less aware of the repetition as the true source of experienced fluency and they may therefore be more likely to misattribute perceived fluency to the $\mathrm{PM}$ intention.

\section{Method}

Participants and design Eighty-nine students participated for course credit or monetary incentives. Six participants who could not recall the PM key or had a zero cue-discrimination performance in a post-experimental intention-memory test (see below) were excluded from the analyses. Participants were randomly assigned to two experimental conditions. In one condition $(n=41)$ there was a lag of five stimuli between the first and second lure presentation, in the other condition $(n=42)$ there was a lag of ten stimuli.
Materials and procedure Prior to the experiment, informed consent and basic demographic information were obtained from all participants. The experiment started with instructions for a lexical-decision task asking participants to press the $A$ key for words and the $L$ key for nonwords. In addition to this ongoing task, participants were asked to form the PM intention to press the 7 key whenever one of the words Taucher (diver), Fenster (window), Nachricht (message), Ausweis (passport), Gerät (device), or Faden (thread) occurred. After ten lexical-decision practice trials, participants performed a 5min filler task (i.e., a figural reasoning task, Raven, 1962). For this task, participants were presented with figural patterns in which one piece was missing. Participants had to select the piece that would complete the pattern out of a set of eight pieces, which were presented right below the pattern, by using the number keys. Then, participants performed 224 trials of the ongoing task with the embedded PM task. On each trial of this task, participants were presented with a letter string until they made a response. For each string, participants had to decide whether it was a word or not. On all trials, strings were preceded by a fixation cross presented for $150 \mathrm{~ms}$. Consecutive trials were separated by a 1,500-ms interstimulus interval. As in our previous studies with the same task, words of medium frequency and length were taken from a German word-norm database. Nonwords were created by jumbling letter pairs in a subset of the words (for details see Meiser \& Schult, 2008). Half of all trials were word trials (including the six PM trials) the other half were nonword trials. Words and nonwords were randomly assigned to trials with the following constraints. PM cues were always presented on Trials 31, 73, 104, 141, 181, and 220. Stimuli on Trials 64, 125, 163, and 212 were predetermined words (i.e., Nagel [nail], Maschine [machine], Rauch [smoke], or Prüfung 
[test]). In one condition (lag5), these words were identical to the words presented five trials before. In the other condition (lag10), these words were identical to the words presented ten trials before. After having performed this task, participants were asked to recall the PM key and to identify the PM cues out of a sequence of stimuli. In the test sequence, the six target cues of the PM task, the four lures, four neutral words from the ongoing task, as well as six new words that had not been presented during the experiment were selected as probe words. However, due to a programming error, each participant was only presented with a random subset of 12 stimuli out of the 20 probes in Experiment 1. Participants were asked to press the $A$ key for cues and the $L$ key for all other words, to assess to which extent they would have been able to recognize them during the ongoing task. After the cue recognition test, participants were debriefed and dismissed.

\section{Results}

Ongoing-task performance Error rates (i.e., percentage of words classified as nonwords or vice versa) and response times of the ongoing lexical-decision task were compared between the lag5 and the lag10 conditions. To control for aftereffects of PM (Meier \& Rey-Mermet, 2012) the four trials following a PM cue or lure (as well as the first four trials of the task) were excluded from all ongoing-task-performance analyses in the present experiments. Means and standard errors of ongoing-task error rates and response times are presented in Table 2.

Ongoing-task error rates did not vary with lag condition, $|t|$ $<1$. Ongoing-task response times (RTs) were calculated for accurate responses only. Because word RTs should better reflect PM processes in situations where the PM cues are words (Brewer, 2011), analyses were confined to word trials. However, considering nonwords in a within-subjects design would not have changed the results of any of the studies reported here. As in our previous studies (e.g., Rummel \& Meiser, 2013), responses faster than $300 \mathrm{~ms}$ or slower than 2 standard deviations (SDs) above an individual's mean RT were discarded as outliers. Word RTs did not vary with lag condition, $|t|<1.2$.

Taken together, these findings imply that ongoing-task performance was not affected by the lag manipulation.

Correct prospective memory (PM) responses The percentage of 7-key presses in response to PM cues was calculated as a measure of correct PM responses and compared between the two conditions (see Table 3 for means). Thereby, 7-key presses on PM trials or the trials directly following a PM trial were counted as correct. The rate of correct PM responses did not vary with lag condition, $|t|<1$, and was similar to previous studies using a similar task (e.g., Wesslein, Rummel, \&
Boywitt, 2014). Thus, the presentation of lure primes seemed to have hardly any effect on the rate of correct PM responses.

False PM responses False PM-response rates reflect the percentage of 7-key presses on trials which were not PM trials. False PM-response rates were calculated for neutral trials and for lure trials (including the trials directly following a lure trial) separately. Because the critical stimuli were discrepant only after their first presentation, the second presentations of repeatedly presented stimuli were considered lure trials. Mean false response rates are presented in Table 3.

To determine whether false PM response rates were increased for words of unexpected processing fluency they were submitted to a $2 \times 2$ mixed analysis of variance (ANOVA) with the between-subjects factor lag (lag5, lag10) and the within-subjects factor stimulus type (neutral, lure). The ANOVA revealed significant main effects of lag, $F(1,81)=$ $6.25, p=.014, \eta_{\mathrm{p}}^{2}=.072$, and stimulus type, $F(1,81)=6.92$, $p=.006, \eta_{\mathrm{p}}^{2}=.089$. These effects were further qualified by a significant interaction, $F(1,81)=5.85, p=.018, \eta_{\mathrm{p}}^{2}=.067$. Simple main effects revealed that there was a significant increase in false PM responses to lures compared to neutral words in the lag10 condition, $F(1,81)=13.81, p<.001, \eta_{\mathrm{p}}^{2}$ $=.146$, but not in the lag 5 condition, $F<1$. Thus, the results of the lag 10 condition corroborated our hypothesis that an unexpected processing-fluency experience can reactivate currently held intentions and serve as a trigger for intention execution. No evidence for such an effect was found in the lag 5 condition.

Importantly, only $2 \%$ of all participants of the lag 5 condition but $19 \%$ of the participants of the lag 10 condition showed at least one false PM response to a lure during the experiment. This finding corroborates that there were substantial differences in the frequency of the occurrence of false PM responses to lures between conditions and that the effect in the lag10 condition was not driven by just a few participants who committed all false responses.

Intention-memory test All but the two excluded participants recalled the PM key. Performance in the post task cuerecognition test was compared between the two lag conditions (see Table 4 for descriptive values). ${ }^{1}$ There was no significant effect of lag condition on cue hit rates (i.e., the percentage of cues classified correctly in the final recognition test), on cue false-alarm rates (i.e., the percentage of words incorrectly classified as cues), and on the cue-discrimination index $\mathrm{Pr}$ (i.e., $\operatorname{Pr}=$ cue hit rate - cue false-alarm rate), all $|t| \mathrm{s}<1$. Across conditions, $\operatorname{Pr}$ was positively correlated with the rate of correct PM responses, $r(83)=.473, p<.001$, but not with

\footnotetext{
${ }^{1}$ As participants were exposed to random subsets of stimuli in this test, hit and false alarm-rates were computed based on the actual frequencies of cue and non-cue occurrences.
} 
Table 2 Ongoing-task error rates and response times

\begin{tabular}{|c|c|c|c|c|c|c|}
\hline & \multicolumn{2}{|c|}{ Error rate $(\%)$} & \multicolumn{2}{|c|}{ Word RTs (ms) } & \multicolumn{2}{|c|}{ Nonword RTs (ms) } \\
\hline & $M$ & $S E$ & $M$ & $S E$ & $M$ & $S E$ \\
\hline \multicolumn{7}{|l|}{ Experiment 1} \\
\hline $\operatorname{Lag} 5(n=41)$ & 7.92 & 1.10 & 794 & 22 & 835 & 31 \\
\hline $\operatorname{Lag} 10(n=42)$ & 7.63 & 0.81 & 754 & 29 & 790 & 22 \\
\hline \multicolumn{7}{|l|}{ Experiment $2 \mathrm{a}$} \\
\hline Short cue-list $(n=36)$ & 5.18 & 0.76 & 918 & 29 & 964 & 32 \\
\hline Long cue-list $(n=37)$ & 6.74 & 0.89 & 893 & 33 & 943 & 36 \\
\hline \multicolumn{7}{|l|}{ Experiment $2 b$} \\
\hline High lure frequency $(n=38)$ & 3.73 & 0.41 & 940 & 34 & 1009 & 38 \\
\hline Experiment 3 & & & Match & & Nonm & \\
\hline Short cue-list $(n=30)$ & 10.83 & 1.21 & 1415 & 84 & 1535 & 95 \\
\hline Long cue-list $(n=30)$ & 13.10 & 1.86 & 1407 & 86 & 1521 & 102 \\
\hline
\end{tabular}

Note. Error rates in the ongoing task in percent. Mean response times (RTs) to words and nonwords or matching and nonmatching colors in the ongoing task are given in ms. Lag conditions (lag5, lag10) denote the number of neutral trials between the first and the second presentation of lure stimuli in Experiment 1.

the rate of false PM responses to lures, $r(83)=-.032, p=.774$, suggesting that the false responses to lures were not primarily committed by those participants who had poor cue memory. Similar correlational patterns were found when the two conditions were analyzed separately.

We also conducted an ANOVA of false alarms to neutral and lure stimuli that appeared in the ongoing task. The $2 \times 2$ ANOVA with the between-subjects factor lag (lag5, lag10) and the withinsubjects factor stimulus type (neutral, lure) yielded a main effect of stimulus type, $F(1,84)=6.81, p=.011, \eta_{\mathrm{p}}^{2}=.078$ with higher false-alarm rates to lures $(M=14.37 ; S E=2.80)$ than to neutral ongoing-task words $(M=7.37 ; S E=2.19)$. The main effect of lag condition and the interaction were not significant, both $F_{\mathrm{S}}<1$.

\section{Discussion}

Results of Experiment 1 provide empirical support for our hypothesis that an unexpected processing-fluency experience can reactivate unfulfilled intentions. False PM-response rates to fluent lures compared to non-fluent neutral stimuli were increased in the lag10 condition. This finding is further empirical support for a PM process which makes use of the

Table 3 Prospective-memory response rates

\begin{tabular}{|c|c|c|c|c|c|c|}
\hline & \multicolumn{2}{|c|}{ Correct responses (\%) } & \multicolumn{4}{|c|}{ False responses (\%) } \\
\hline & \multicolumn{2}{|c|}{ Target cues } & \multicolumn{2}{|c|}{ Lures } & \multicolumn{2}{|c|}{ Neutral stimuli } \\
\hline & $M$ & $S E$ & $M$ & $S E$ & $M$ & $S E$ \\
\hline \multicolumn{7}{|l|}{ Experiment 1} \\
\hline $\operatorname{Lag} 5(n=41)$ & 47.56 & 5.03 & 0.61 & 0.61 & 0.20 & 0.07 \\
\hline $\operatorname{Lag} 10(n=42)$ & 53.17 & 4.18 & 5.95 & 2.05 & 0.42 & 0.02 \\
\hline \multicolumn{7}{|l|}{ Experiment $2 \mathrm{a}$} \\
\hline Short cue-list $(n=36)$ & 59.72 & 5.03 & 1.85 & 1.45 & 0.15 & 0.08 \\
\hline Long cue-list $(n=37)$ & 44.59 & 4.88 & 3.15 & 1.42 & 0.89 & 0.34 \\
\hline \multicolumn{7}{|l|}{ Experiment $2 b$} \\
\hline High lure frequency $(n=38)$ & 48.68 & 5.42 & 1.77 & 0.65 & 0.56 & 0.23 \\
\hline \multicolumn{7}{|l|}{ Experiment 3} \\
\hline Short cue-list $(n=30)$ & 40.67 & 5.87 & 0.33 & 0.33 & 0.65 & 0.29 \\
\hline Long cue-list $(n=30)$ & 30.67 & 5.60 & 5.67 & 2.13 & 1.67 & 0.82 \\
\hline
\end{tabular}

Note. Prospective memory (PM) response rates to cues, to lures (i.e., stimuli whose processing fluency was increased), and to neutral ongoing-task stimuli in percent. Lag conditions (lag5, lag10) denote the number of neutral trials between the first and the second presentation of lure stimuli in Experiment 1. 
Table 4 Cue-recognition rates

\begin{tabular}{|c|c|c|c|c|c|c|}
\hline & \multicolumn{2}{|c|}{ Cue HR (\%) } & \multicolumn{2}{|c|}{ Cue FAR (\%) } & \multicolumn{2}{|c|}{ Cue $\operatorname{Pr}(\%)$} \\
\hline & $M$ & $S E$ & $M$ & $S E$ & $M$ & $S E$ \\
\hline \multicolumn{7}{|l|}{ Experiment 1} \\
\hline $\operatorname{Lag} 5(n=41)$ & 84.31 & 3.63 & 8.74 & 2.22 & 75.57 & 4.18 \\
\hline $\operatorname{Lag} 10(n=42)$ & 86.71 & 2.82 & 6.46 & 2.27 & 80.24 & 3.68 \\
\hline \multicolumn{7}{|l|}{ Experiment $2 \mathrm{a}$} \\
\hline Short cue-list $(n=36)$ & 95.37 & 1.43 & 6.02 & 2.91 & 89.35 & 3.16 \\
\hline Long cue-list $(n=37)$ & 45.50 & 2.56 & 3.15 & 0.99 & 42.34 & 2.51 \\
\hline \multicolumn{7}{|l|}{ Experiment $2 b$} \\
\hline High lure frequency $(n=38)$ & 82.68 & 2.64 & 7.68 & 1.65 & 75.00 & 3.14 \\
\hline \multicolumn{7}{|l|}{ Experiment 3} \\
\hline Short cue-list $(n=30)$ & 90.67 & 2.83 & 6.67 & 2.16 & 84.00 & 3.73 \\
\hline Long cue-list $(n=30)$ & 82.33 & 3.92 & 9.67 & 1.95 & 72.67 & 5.07 \\
\hline
\end{tabular}

Note. Hit rates (Cue HR) and false-alarm rates (Cue FAR) of the cue-recognition test applied after the task in percent. Cue Pr= Cue HR - Cue FAR. Lag conditions (lag5, lag10) denote the number of neutral trials between the first and the second presentation of lure stimuli in Experiment 1.

discrepancy between expected and factual processing experience (McDaniel et al., 2004). There was no fluency effect, however, in the lag5 condition. This might be due to the fact that participants of the lag 5 condition realized that some words were presented repeatedly so that, in contrast to the less optimal lag10 condition, controlled processes were more likely to detect the true source of fluency. In particular, being aware of the fact that the lure words occurred a short time ago enables participants to correctly attribute the processing-fluency experience to the previous occurrence of the word. In a similar vein, Jacoby and Whitehouse (1989) found that false "old" recognition judgments to lures that were fluent due to priming did not occur when participants were aware of the primes (i.e., understood the true source of fluency; see also Bernstein \& Welch, 1991; Joordens \& Merikle, 1992; Klinger, 2001). This may explain why participants of the lag5 condition did not misinterpret the fluent stimuli as a signal to execute the intended action.

While the findings of Experiment 1 demonstrate that unexpectedly fluent processing can sometimes initiate a false intention execution, it is not clear whether the origin of this effect is completely spontaneous in nature. As participants already saw the lure words during their first presentation, it might be that participants confused the sources of lure fluency in episodic memory. That is, they may have been well aware that they were presented with the lure words before, but they falsely assumed that they remembered the words from the PM cue list. If false PM responses to lures were due to this kind of source confusion, they would not necessarily reflect a spontaneous process but could mirror controlled PM processes on the basis of episodic source-memory failures. The finding of higher false-alarm rates to lures as compared to neutral items in the intention-memory test is in line with this alternative interpretation. In Experiment 2a, we therefore tested whether false intention execution would also be initiated by unexpectedly fluent processing of stimuli which were not presented in the cue list or in the ongoing task before and which could thus not be confused in episodic memory.

\section{Experiment 2a}

As the increased false PM response rate to lures in Experiment 1 could reflect a consciously operating source (mis-)attribution of fluency in episodic memory, we increased the fluency of the PM lures on the spot and in a more subtle manner in Experiment 2a. For this purpose, we employed an identitypriming procedure. Again, we hypothesize that an unexpected fluency experience will spontaneously reactivate unfulfilled intentions. Thus, we expected false PM-response rates to be increased for fluently processed lures, even when the lures were hitherto as familiar as the other stimuli in the task environment. Moreover, as outlined above, we assume that the reliance on fluency experiences will be especially high under conditions that are suboptimal for a successful controlled intention-memory search (Moors \& De Houwer, 2006b). In line with this idea, it has been shown, for example, that individuals especially rely on fluency experiences for recognition memory judgments in stages of memory uncertainty (Johnston et al., 1991). Therefore, we also manipulated the length of the cue list to investigate whether false responses to lures become more likely under increased cue-memory uncertainty. This manipulation was based on previous research showing that a long as compared to a short PM cue list negatively affects PM performance as well as cue memory (A.-L. Cohen, Jaudas, \& Gollwitzer, 2008; Wesslein et al., 2014).

For exploratory reasons, half of the participants in each condition of the present experiment were also asked to 
respond to PM cues with the PM key directly, while the other half were asked to first make a lexical decision and then the PM response. Additionally, for half of the participants in each experimental condition we not only primed the lures but also the cues.

\section{Method}

Participants and design Seventy-six students participated for course credit. One participant with zero cue-discrimination performance in the post-task recognition test and two participants who did not follow the ongoing-task instructions were excluded. The remaining participants were randomly assigned to a short $(n=36)$ and a long $(n=37)$ cue-list condition.

Materials and procedure Materials and procedure were identical to the ones in Experiment 1 with the following exceptions: Participants of both the long and the short cue-list conditions performed 210 trials of the ongoing lexicaldecision task (half word, half nonword trials) with the embedded PM task. For the ongoing task, participants had to press the $A$ key for words and the $L$ key for nonwords. On each trial, a 200-ms fixation cross was followed by a forward mask, a prime, and a backward mask. Masks were letter strings of ten $\mathrm{Xs}$ and the primes were meaningless random-letter strings in most cases. Only on lure trials (and also on PM trials for half of the participants in each cue-list length condition), the primes were identical to the succeeding word. Masks and primes were presented for $54 \mathrm{~ms}$ each. Prime duration was aligned to previous research showing that an identity priming of $40 \mathrm{~ms}$ for cues improves PM performance (Graf, 2005). The slightly longer duration in the present study was chosen for technical reasons to adjust presentation times to the refreshing cycles of the computer screens. Prime presentation durations of similar length yielded an optimum level of unconscious influences on subsequent perceptual processing in previous studies (Meier, Morger, \& Graf, 2003).

All participants learned that they should respond with the $U$ key to the words Fenster (window), Nachricht (message), Karton (box), Winter (winter), Rauch (smoke), and Nagel (nail) when they occurred during the ongoing task. Half of the participants in each condition were instructed to press this key in addition to the word/nonword classification, the other half were asked to press this key instead of making a word/ nonword classification. Participants in the long cue-list condition associated the intention with six additional words (i.e., Ameise [aunt], Restaurant [restaurant], Taucher [diver], Prüfung [test], Machine [machine], and Sessel [armchair]) that were not presented during the ongoing task. For all participants, PM cues occurred on Trials 25, 55, 91, 125, 157, and 189. Six lure words were selected randomly and for each participant anew from the body of 105 word stimuli of the ongoing task. Lures (i.e., words preceded by an identity prime) were presented on Trials $42,74,106,140,176$, and 208 . For the final intention-memory test, participants were asked to recall the PM key and to identify the cues in a test sequence. For this sequence, all participants were presented with all cue words (six or 12, respectively) and 12 distractor words in random order. Distractor words were six neutral words from the ongoing task and six new words that had not been presented during the experiment. After the task, participants were asked whether they had noticed the presentation of words during the masked priming procedure preceding the onset of the ongoing-task stimuli.

\section{Results}

As the manipulation of whether participants should respond to cues immediately or after having made a lexical-decision first did not affect the present data in any way, we collapsed across this factor for all analyses. Whereas the statistical power to detect a medium-sized fluency effect within each group was sufficiently high $(1-\beta>.80)$, the power to detect betweensubjects effects of list length and cue priming of medium size was lower $(1-\beta=.58)$.

Ongoing-task performance Ongoing-task error rates and RTs (trimmed as before) did not vary with cue-list length, $|t|$ $<1.4$, (see Table 2).

Correct PM responses A $2 \times 2$ ANOVA with the betweensubjects factors cue-list length (short, long) and cue priming (yes, no) for correct PM response rates only revealed a significant effect of list length. In line with our previous research (Wesslein et al., 2014), correct PM rates were higher under short cue-list than under long cue-list conditions, $F(1,69)=$ 4.72, $p=.033, \eta_{\mathrm{p}}^{2}=.064$ (see Table 3). Whether cues were preceded by an identity prime $(M=52.94 ; S E=4.95)$ or not $(M=50.56 ; S E=4.89)$, however, did not affect the rate of correct PM responses, $F<1$. The interaction was also not significant, $F(1,69)=3.14, p=.081, \eta_{\mathrm{p}}^{2}=.043$. As the cue-priming manipulation did not affect any other result of the present experiment, we collapsed across this manipulation for all other analyses.

False PM responses The $2 \times 2$ mixed ANOVA with the between-subjects factor cue-list length (short, long) and the within-subjects factor word type (neutral, lure) for false PMresponse rates yielded a main effect of word type, $F(1,71)=$ $4.56, p=.036, \eta_{\mathrm{p}}^{2}=.060$, indicating higher rates of false PM responses to primed lures than to neutral stimuli (see Table 3 ). The main effect of cue-list length and the interaction were not significant, both $F_{\mathbf{S}}<1$. Thus, in line with our hypothesis that a processing-fluency experience spontaneously reactivates unfulfilled intentions, the presentation of lures again caused an increase in false PM responses. This increase, however, 
was not moderated by cue-list length. In total, $7 \%$ of all participants in the short-list and $14 \%$ of all participants in the long-list condition showed at least one false PM responses to a lure. Thus, several participants were sensitive to the fluency manipulation and the proportion of sensitive participants was descriptively higher in the long-list than in the short-list condition.

Fourteen percent of all participants stated that they had noticed the identity primes when they were explicitly asked whether they had seen them or not. Importantly, the pattern of means remained largely unchanged when analyzing only those participants who indicated that they did not notice the primes, although the statistical effect of word type was only marginally significant due to the reduced statistical power.

Intention-memory test All participants were able to recall the PM key. Cue-hit rates were significantly higher in the short compared to the long cue-list condition, $t(71)=19.19, p<$ $.001, d=4.55$ but false-alarm rates did not vary with cue-list length, $|t|<1$. The cue-discrimination index $\operatorname{Pr}$ also reflected better target discrimination in the short cue-list condition than in the long cue-list condition, $t(71)=11.68, p<.001, d=2.77$ (cf. Table 4). Across conditions, $\operatorname{Pr}$ was positively correlated with the proportions of correct PM responses, $r(73)=.291, p$ $=.012$ and, unlike in Experiment 1, negatively correlated with the proportion of false PM responses to lures, $r(73)=-.256, p$ $=.029$. Similar correlational patterns were found when the two conditions were analyzed separately. This finding suggests that the false responses to lures were primarily committed by those participants who had poor cue memory. However, as the total percentage of participants committing at least one false PM response was rather low in this experiment, the correlation between cue-discrimination and PM-false-response rate has to be interpreted with caution. In fact, to preview results from the following two experiments, we did not find further evidence for such a correlation in the other studies reported here.

\section{Discussion}

The finding that false PM responses to lures were higher than to neutral ongoing-task stimuli further supports the hypothesis that an unexpected fluency experience can reactivate intentions and can trigger an erroneous intention execution. As fluency was increased via identity priming of a randomly chosen subset of ongoing-task words, this finding cannot be due to differences in the familiarity of lures and neutral words, caused by prior presentation and episodic-memory confusion. Furthermore, as the assignment of words to lure and neutral trials was randomly determined for each participant anew, it can be ruled out that pre-experimental associations between $\mathrm{PM}$ cues and lure stimuli were responsible for the observed increase in false PM responses to lures. We assumed that individuals will rely on an experience of processing fluency especially in the case of cue-memory uncertainty. In fact, there were more participants in the long-list than in the short-list condition which responded to lures with a false PM response but the increase in false PM responses to lures from the short to the long cue-list condition was not statistically reliable. Additionally, cue-list length negatively affected cue recognition but did not affect false-alarm rates in the post task cuerecognition test. Thus, our hypothesis that fluency-based spontaneous intention reactivation should become more likely with increased cue-list length was not supported by the present findings.

Whereas the priming manipulation resulted in an increase in false PM responses to lures, rates of correct responses to PM cues were not affected by the fact of whether the cues were preceded by an identity prime or not. In the light of a previous study that found PM improvements due to cue identity priming (Graf, 2005), this finding seems surprising at first glance, but it is well in line with the fluency literature in recognition memory which has repeatedly shown that fluency has stronger effects on false responses to lures than on correct responses to targets (Joordens \& Merikle, 1992; Kurilla \& Westerman, 2008). As the number of cues was quite high in the present study, it might well be that PM-cue detection strongly relied on controlled PM processing (see also A.-L. Cohen et al., 2008) and that the influence of the subtle fluency manipulation on correct PM response rates was thus negligible.

To replicate and extend the main finding that an unexpected fluency experience can spontaneously reactivate intentions and trigger false intention execution and to investigate the robustness of this effect, we ran another experiment with a larger number of lures.

\section{Experiment 2b}

Experiment $2 \mathrm{~b}$ was similar to the long cue-list condition of Experiment $2 \mathrm{a}$ except that one-third instead of only $5 \%$ of all ongoing-task words were preceded by an identity prime. As there are more opportunities to respond to a lure with a false PM response when there are more lure trials, we expected that the proportion of participants who responded to a lure with a PM response would be generally higher in this experiment than in Experiment 2a. Moreover, assuming that processing fluency reliably elicits false PM responses on only few instances even when lures occur more frequently, the increased number of lures enabled us to analyze response times of correct responses in the ongoing task to lures. In doing so, we aimed to shed further light on the question of how the fluent stimuli are processed. If fluent lures were generally attended to and then correctly rejected as being not a PM cue, one would expect that the processing of lures is slowed compared to neutral stimuli. Alternatively, if extra attention to lures reflects 
a more incidental phenomenon that leads to false PM responses in some cases, one would not expect that correct responses lures are generally slowed. Last but not least, expecting a typical priming effect, one could even assume responses to lures to be faster than to neutral stimuli.

\section{Method}

Participants and design Forty students participated for course credit. One participant with zero cue-discrimination performance in the final recognition test and one participant who did not follow the ongoing-task instructions were excluded, resulting in $N=38$ participants.

Materials and procedure Materials were the same as in Experiment $2 \mathrm{a}$ and the procedure was identical to the long cue-list procedure of Experiment $2 \mathrm{a}$ with the following exceptions: Primes and masks were presented for $40 \mathrm{~ms}$. Presentation duration of primes and masks was shortened relative to Experiment $2 \mathrm{a}$, because the computer screens used for Experiment $2 \mathrm{~b}$ had a different refreshing cycle. This allowed for an even more subtle priming manipulation. The number of lures was increased so that in one-third of all word trials of the ongoing lexical-decision task, the word was preceded by an identity prime. In total, there were six PM trials, 33 lure trials, 66 neutral word trials, and 105 nonword trials. Trials were randomly mixed for each participant anew but PM cues were presented on fixed positions, that is, on Trials 42, 74, 106, 137 , 170 , and 202.

\section{Results}

Ongoing-task performance Ongoing-task error rates and RTs were comparable to previous studies (see Table 2).

Correct PM responses The rate of correct PM responses was comparable to the long-list condition of Experiment 2a (see Table 3).

False PM responses Replicating Experiment 2a, the pairedsample $t$-test comparing false PM-response rates to lure words and to neutral words revealed a higher rate of PM responses to lures than to neutral words, $t(37)=2.12, p=.041, d_{z}=0.57$ (see Table 3).

This time, $21 \%$ of all participants showed at least one false PM response to a lure. Thus the proportion of participants who were sensitive to the lure manipulation was higher than in Experiment $2 \mathrm{a}$ and as high as in Experiment 1 where a less subtle repetition priming procedure was used. Four percent of all participants indicated that they saw the primes when they were asked after the experiment. Analyzing only those participants who stated that they did not notice the primes showed the same pattern of means but rendered the statistical effect only marginally significant due to the reduction of statistical power.

Response times to lures RTs were trimmed as before separately for lure words and neutral words. By comparing RTs to correctly classified lures and RTs to correctly classified neutral words, we found a typical priming effect, that is, lure RTs $(M=892 ; S E=37)$ were faster than neutral-word RTs $(M=940 ; S E=34) ; t(37)=3.50, p=.001, d_{z}=0.34$.

Intention-memory test All participants were able to recall the PM key. Cue recognition was comparable to the long cue-list condition of Experiment 2a (see Table 4). Pr was positively correlated with the proportions of correct PM responses, $r(38)=.394, p=.014$, but not significantly correlated with the proportion of false PM responses to lures, $r(38)=-.212$, $p=.202$.

\section{Discussion}

In Experiment 2b, we again showed that fluently processed stimuli can trigger false PM responses, which was revealed by an enlarged proportion of false PM responses to lures relative to neutral items. With the higher number of lures, the percentage of participants that responded at least once with a PM response to lures was also higher than in the previous Experiment $2 \mathrm{a}$. Increasing the number of lures in Experiment $2 \mathrm{~b}$ also allowed us to compare RTs to lures and neutral words. In doing so, we found a typical priming effect, that is, correct responses to lures were generally faster than to neutral words. This pattern of results suggests that fluent lures have a general processing advantage. Thus, the processing of lures was not especially time-consuming, suggesting that fluently processed lures were not generally actively attended to and then rejected as potential PM cues. Nonetheless, the fluent lures reliably elicited false intention execution on some occasions. We may therefore conjecture that if a lure captures attention as a potential PM cue, it is likely to sometimes be misattributed as a relevant trigger of the intended action in the context of the PM task.

\section{Experiment 3}

In the previous experiments, we used a lexical-decision task as ongoing task. In Experiment 3 we realized a short and a long cue-list condition similar to the ones in Experiment 2a, but we embedded the PM task in an ongoing color-matching task. On each trial of this task, participants must maintain and update color information and this task should thus impose additional working-memory demands on participants (cf. Redick \& Lindsey, 2013). The aim of this study was twofold. On the one hand, we aimed to replicate results from Experiment $2 \mathrm{a}$ 
with other materials. On the other hand, the more demanding ongoing task also allowed us to better test the hypothesis that spontaneous processes especially prevail under processing conditions that are suboptimal for controlled processing (Moors \& De Houwer, 2006a, 2006b). This time, suboptimal conditions were realized by the combination of an attentiondemanding ongoing task, which should reduce the attentional resources available for controlled PM processing (Harrison, Mullet, Whiffen, Ousterhout, \& Einstein, 2013; Marsh, Hancock, \& Hicks, 2002; McDaniel et al., 2004) with a manipulation of cue-memory load. In line with Moors and De Houwer (2006a), we assumed that fluency-induced false intention execution will be especially high under these conditions. Alternatively, however, one could argue that fluencyinduced intention reactivation will be reduced under these conditions, because individuals get more absorbed by a demanding ongoing task and the fluent lures are therefore not sufficiently processed to trigger intention retrieval. Recent findings that spontaneous PM processing is hampered by challenging ongoing tasks (Harrison et al., 2013) are in line with this latter assumption. Therefore, it remains an open question whether an unexpected experience of fluency will still reactivate unfulfilled intentions, when the ongoing task is attention demanding.

\section{Method}

Participants and design Sixty students participating for course credit or monetary compensation were randomly assigned to a short $(n=30)$ and a long $(n=30)$ cue-list condition.

Materials and procedure Materials and procedure were similar to Experiment 2a but we used a letter-task as a filler task and a color-matching task as an ongoing task. At each trial of the letter task, participants were presented with a letter (randomly chosen from all letters of the alphabet, excluding the letters A and B) and had to press the letter on the keyboard which alphabetically precedes the currently presented letter by two positions. Letters were presented on the screen until a response was given. At each trial of the color-matching task, participants were presented with a series of four colored squares followed by a word that could have either the same color as one of the preceding squares or a different one (adapted from Smith \& Bayen, 2004; see also Rummel, Boywitt, \& Meiser, 2011, for a detailed description of task materials). Words of medium frequency and length were drawn from a German word-norm database. For each word, participants had to decide whether its color matched one of the preceding squares or not by pressing the $J$ or the $N$ key, respectively.

For the PM task, participants were asked to press the 1 key whenever one of the words Dorf (village), Leute (people),
Haltung (attitude), Boot (boat), or Fenster (window) occurred during the ongoing task. For participants in the long cue-list condition, the words Abend (evening), Status (status), Ausdruck (expression), Nähe (proximity), and Vorjahr (previous year) were additionally encoded as cues, but participants in this condition were also only presented with a randomly chosen subset of five cue words during the ongoing task. All participants performed 150 trials of the ongoing colormatching task (half match, half nonmatch trials) and PM cues always occurred on Trials 20,45, 80, 110, and 140. Trial type (match versus nonmatch trials) was randomly determined. Words were randomly assigned to trials for each participant anew. Neutral ongoing-task words and PM cues were preceded by meaningless random letter strings that were presented for $42 \mathrm{~ms}$ and covered by forward and backward masks (see Experiment 2a). Lures were created via identity priming of the words assigned to Trials 15, 35, 50, 60, 70, 90, 100, 125, 134, and 148 , with trial type being randomly determined. After the task, participants were asked to recall the PM key and to identify the PM cues in a recognition test consisting of the cues and ten distractor words. Half of the distractor words were words from the ongoing task, the other half were new words. Finally, participants were asked whether they saw the primes on lure trials or not.

\section{Results}

Ongoing-task performance Means and standard errors of ongoing-task error rates and RTs are presented in Table 2. Ongoing-task error rates did not vary with cue-list length, $|t|$ $<1.1$. RTs on match and non-match trials were trimmed separately applying the same criteria as before. As PM targets could occur on match and on nonmatch trials, RTs of both trial types should reflect PM processes and were thus analyzed together. The $2 \times 2$ mixed ANOVA with the betweensubjects factor cue-list length (short, long) and the withinsubjects factor trial type (match, nonmatch) for RTs yielded a main effect of trial type, $F(1,58)=81.08, p<.001, \eta^{2}=$ .583 , with shorter RTs for match than nonmatch trials. The main effect of cue-list length and the interaction were not significant, both $F \mathrm{~s}<1$. Thus, as in the Experiment 2 a, ongoing-task performance was not affected by the cue-list length manipulation.

Correct PM responses The rate of correct PM responses did not differ between the short and the long cue-list conditions, $t(58)=1.20, p=.237, d=0.32$ (see Table 3$)$.

False $P M$ responses $A 2 \times 2$ mixed ANOVA with the between-subjects factor cue-list length (short, long) and the within-subjects factor stimulus type (neutral, lure) for false PM-response rates yielded a main effect of list length, $F(1$, $58)=5.20, p=.026, \eta_{p}^{2}=.082$ and a main effect of stimulus 
type, $F(1,58)=4.50, p=.038, \eta_{\mathrm{p}}^{2}=.072$. The main effects were qualified by a significant interaction, $F(1,58)=6.18, p=$ $.016, \eta_{\mathrm{p}}^{2}=.096$. Simple-effect analyses revealed a significantly increased false-response rate to lures compared to neutral stimuli in the long cue-list condition, $F(1,58)=10.60, p=$ $.002, \eta_{\mathrm{p}}^{2}=.155$, but not in the short cue-list condition, $F<1$ (see Table 3).

In total, only $3 \%$ of participants of the short-list but $27 \%$ of participants of the long-list condition showed at least one false PM response. Notably, the percentage of participants who committed one or more false responses in the long-list condition was even higher than in Experiment 1. Ten percent of all participants indicated that they saw the lure primes. Analyzing only those participants who stated that they did not notice the primes did not change the results in any way.

Intention-memory test All participants were able to recall the PM key. Cue-hit rates were marginally higher in the short compared to the long cue-list condition, $t(58)=1.72, p=$ $.090, d=0.45$. False-alarm rates did not vary with cue-list length, $|t|<1.1$. The cue-discrimination index $\operatorname{Pr}$ also showed a non-significant trend of better cue discrimination in the short than in the long cue-list condition, $t(58)=1.80, p=.077, d=$ 0.47 (see Table 4). Across conditions, $\operatorname{Pr}$ was marginally correlated with the proportion of correct PM responses, $r(60)=$ $.242, p=.062$, but uncorrelated with the proportion of false PM responses to lures, $r(60)=-.078, p=.551$. Similar correlational patterns were found within each condition. Thus, there was no evidence that the false responses to lures were primarily committed by those participants who had poor memory for the PM cues.

\section{Discussion}

As in Experiment 2a, participants of Experiment 3 were assigned to a short and a long cue-list condition but a different ongoing task was applied. The ongoing color-matching task supposedly imposed higher working-memory demands on the individual than the lexical-decision task used in Experiment 2a, because, in order to succeed in this task, participants had to maintain the colors of the squares in short-term memory and also to update the colors on each trial.

In Experiment 3, cue-list length moderated the increase in false PM responses to lures. Only with a long cue list, participants responded with a PM response more frequently to lures than to neutral stimuli. Thus, the lure-induced increase in false PM responses was less stable than in the previous studies with less demanding ongoing tasks. This result may indicate that spontaneous $\mathrm{PM}$ processing is not completely automatic or/and requires full processing of the PM cues (and lures), which can be hampered by demanding ongoing tasks (Harrison et al., 2013). Importantly, the present pattern of results is also in line with our assumption that suboptimal PM processing conditions (i.e., high cue-memory uncertainty, in combination with a challenging ongoing task, which draws on attentional resources) render a reliance on spontaneous processing especially likely (cf. Moors \& De Houwer, 2006a, 2006b). In Experiment 2a, the number of participants who were sensitive to the fluency manipulation was numerically higher in the long than in the short cue-list condition. However, unlike in Experiment 3, differences in lure-induced false responses between short and long cue-list conditions were not statistically reliable in Experiment 2a. Thus, further research is necessary to test this hypothesis.

\section{General discussion}

The overarching aim of the present research was to test whether a processing experience that differs from expectancies (i.e., an unexpectedly fluent processing of a hitherto neutral stimulus) spontaneously reactivates intentions. In four experiments, we tested the hypothesis by presenting participants with lures which had never been associated with an intention but whose processing felt discrepant from the ongoing task environment due to increased fluency.

In Experiment 1, lure fluency was manipulated via repetition priming and results showed that false PM responses to lures occurred more frequently than to neutral ongoingtask stimuli. In line with the assumption that fluency experiences affect memory judgments when people are unaware of the true source of fluency (Bernstein \& Welch, 1991; Jacoby \& Whitehouse, 1989; Klinger, 2001), the fluency effects only occurred under spaced repetition conditions with a lag of ten but not with a lag of five. To test whether it is a necessary condition for fluency-based PM processing that participants are consciously aware that they saw the lure stimuli before, we ran three experiments in which lure fluency was increased via more subtle identity priming. In Experiment 2a, false PM responses were triggered by lures, independent of cue-list length. Experiment $2 \mathrm{~b}$ also showed a lure-induced increase in false PM responses and a generally faster processing of fluent lures compared to neutral stimuli. Finally, with the more demanding ongoing task in Experiment 3, the false PMresponse rate to lures compared to neutral stimuli was increased under long cue-list but not under short cue-list conditions. This finding nicely parallels with findings from the recognition memory literature showing that the reliance on fluency is especially high when item memory is poor (Johnston et al., 1991). Results of Experiment 3 are also well in line with the general claim that automatic processes prevail under suboptimal processing conditions (Moors 
\& De Houwer, 2006a, 2006b). Given that cue-list length did not significantly moderate the fluency effect in Experiment 2a, however, further evidence is needed to bolster this assumption. Importantly, we found evidence for fluency-based erroneous intention execution across four experiments. $^{2}$

This finding is of theoretical interest, because it sheds light on the nature of discrepancy-based PM processing. Previous research consistently found PM improvements with increased cue-discrepancy (Breneiser \& McDaniel, 2006; Graf, 2005; Guynn \& McDaniel, 2007; Lee \& McDaniel, 2013; McDaniel et al., 2004). However, these PM improvements need not necessarily reflect a spontaneous process. Alternatively, PM improvements could have been due to a more efficient controlled PM process. It might be, for example, that discrepant cues are easier to detect than nondiscrepant cues for individuals if (and only if) they engage in attentional cue-monitoring (cf. McDaniel \& Einstein, 2007). One way to rule out that controlled monitoring processes are involved in cue-discrepancy-based PM benefits would be to create conditions under which participants are discouraged from monitoring by distracting their attention (Guynn \& McDaniel, 2007). However, it is difficult to ensure that there are virtually no attentional resources left for controlled PM processing. Another possibility would be suspending the intention so that there will be temporarily no need to engage in cue-monitoring and to investigate whether the cues are still noticed (Einstein et al., 2005; Rummel, Einstein, \& Rampey, 2012). Such a task setting presumably allows for isolating spontaneous PM processes. Yet it is also somewhat limited, because in many PM situations both controlled and spontaneous PM processes act together to allow for successful intention fulfillment (see Meiser \& Rummel, 2012). In the present studies, most participants probably engaged in attentional cuemonitoring. In fact, there is empirical evidence that people will monitor for PM cues, as soon as there is more than one cue associated with an intention (A.-L. Cohen et al., 2008). Therefore, we created a situation in which spontaneous and controlled cue-noticing processes should result in different empirical outcomes. Whereas spontaneous processing should result in a false PM response to unexpectedly fluent stimuli,

\footnotetext{
${ }^{2}$ For full disclosure, we want to mention here that we did not find a significant identity priming effect in a very first study (not reported) with $n=30$ participants we ran in this series. In this experiment, we used an ongoing lexical decision task, a long cue-list (ten words), a prime duration similar to the one in the other studies reported here $(42 \mathrm{~ms})$, but the spacing of the primed words was more regular than in the studies reported here (i.e., there were always 15 trials between two lures). Since this first experiment, we consistently found the identity priming effect in three studies and we conceptually replicated it using a repetition priming paradigm (cf. Experiment 1). As one non-significant result out of five attempts to test a hypothesis is in line with the ratio expected in light of the desirable statistical power of psychological studies (i.e., $1-\beta=.80$, J. Cohen, 1968), we are confident, that this effect is robust and reliable.
}

controlled processing should not, because the fluently processed stimuli had never been associated with the PM intention. Following this rationale, the present finding that unexpected processing fluency increased the likelihood of erroneous intention execution in the absence of a PM cue can be taken as empirical support for the hypothesis that discrepancy-based PM processing occurs rather spontaneously. Alternatively, one could argue that false intention execution could have been due to participants' deliberate use of the fluency experience to perform the intention in the case of cue-memory uncertainty. We cannot completely rule out this alternative interpretation of the present results. However, the present findings showed that false intention-execution rates to fluent stimuli were increased even when cue-memory was generally very good, and that they are largely unrelated to cue-discrimination performance in the cue-memory test (i.e., in all experiments despite Experiment 2a). These results are more in line with our interpretation that false intention executions to fluent lures are manifestations of a spontaneous process. Furthermore, the priming effect in terms of faster correct ongoing-task responses to lures compared to neutral stimuli, which was observed in Experiment 2b, renders it unlikely that lures were regularly attended to in a deliberate way and then rejected as lures on the basis of a controlled process.

The fluent lures in the present experiments were stimuli that could have been PM cues (i.e., they were regular words that occurred in the ongoing task) but they had never been associated with the intended action. Thus, the present empirical demonstration that these lures can elicit a false intention execution, just because their processing differs from the majority of the stimuli, implies that fluency-based PM processes, unlike controlled PM processes (i.e., deliberate cuemonitoring; McDaniel \& Einstein, 2007), work in a more global manner. The merits of a discrepancy-induced intention reactivation are quite obvious. It generally helps individuals to fulfill intentions at the right moment (cf. Lee \& McDaniel, 2013). The potential lack of specificity of this mechanism, however, may also come with a cost. That is, under certain conditions that are suboptimal for controlled PM processing, fluency-based PM processing may cause an inappropriate intention execution in response to a cue that for some reason feels related to a pending intention but is, in fact, not the appropriate cue for this particular intention. Imagine, for instance, you intend to go shopping in a specific supermarket after work, because you want to buy a certain product which is only available there. On your way home, you encounter the sign of the supermarket where you usually do your shopping and you enter it, mainly because seeing this sign, which is a reasonable cue for the intention to buy groceries in most other situations, feels fluent. This illustrates that a global intention retrieval system would probably get along with only few cognitive resources but would also sometimes be error-prone. However, although false intention execution rates were 
significantly increased in the presence of the fluent lures in the present studies, they were still quite low. Probably there are controlled processes that usually prevent us from executing our intentions at the wrong time and in inappropriate situations (see also Meiser \& Rummel, 2012; Scullin et al., 2012, for a similar assumption).

Taken together, the present findings not only provide new empirical support for the spontaneous nature of discrepancybased PM processing (Lee \& McDaniel, 2013) but also suggest that an unexpectedly fluent stimulus has the potential to reactivate intentions, even when it is unrelated to it. Thus, the underlying mechanism might operate in a more general manner than hitherto expected as it makes use of rather general signals from the cognitive system, such as an unexpected fluency experience.

Acknowledgments The research reported in this article was funded by a grant from the German Research Foundation (DFG) to the second author (Grant ME1918/3-1).

\section{References}

Bernstein, I. H., \& Welch, K. R. (1991). Awareness, false recognition, and the Jacoby-Whitehouse Effect. Journal of Experimental Psychology: General, 120, 324-328. doi:10.1037/0096-3445.120. 3.324

Breneiser, J. E., \& McDaniel, M. A. (2006). Discrepancy processes in prospective memory retrieval. Psychonomic Bulletin \& Review, 13, 837-841. doi:10.3758/BF03194006

Brewer, G. A. (2011). Analyzing response time distributions methodological and theoretical suggestions for prospective memory researchers. Journal of Psychology, 219, 117-124. doi:10.1027/ 2151-2604/a000056

Bugg, J. M., \& Scullin, M. K. (2013). Controlling intentions: The surprising ease of stopping after going relative to stopping after never having gone. Psychological Science, 24, 2463-2471. doi:10.1177/ 0956797613494850

Cohen, A.-L., Jaudas, A., \& Gollwitzer, P. M. (2008). Number of cues influences the cost of remembering to remember. Memory \& Cognition, 36, 149-156. doi:10.3758/MC.36.1.149

Cohen, J. (1968). Statistical Power Analysis for the Behavioral Sciences (Vol. 2). New Jersey: Lawrence Erlbaum Associates.

Cowan, N., Elliott, E. M., Saults, J. S., Morey, C. C., Mattox, S., Hismjatullina, A., \& Conway, A. R. A. (2005). On the capacity of attention: Its estimation and its role in working memory and cognitive aptitudes. Cognitive Psychology, 51, 42-100. doi:10.1016/j. cogpsych.2004.12.001

Einstein, G. O., \& McDaniel, M. A. (1990). Normal aging and prospective memory. Journal of Experimental Psychology: Learning, Memory, and Cognition, 16, 717-726. doi:10.1037/0278-7393.16. 4.717

Einstein, G. O., McDaniel, M. A., Thomas, R., Mayfield, S., Shank, H., Morrisette, N., \& Breneiser, J. (2005). Multiple processes in prospective memory retrieval: Factors determining monitoring versus spontaneous retrieval. Journal of Experimental Psychology: General, 134, 327-342. doi:10.1037/0096-3445.134.3.327

Graf, P. (2005). Prospective memory retrieval revisited. In N. Ohta, C. M. MacLeod, \& B. Uttl (Eds.), Dynamic cognitive processes. Tokyo, Japan: Springer.
Guynn, M. J., \& McDaniel, M. A. (2007). Target preexposure eliminates the effect of distraction on event-based prospective memory. Psychonomic Bulletin \& Review, 14, 484-488. doi:10.3758/ BF03194094

Harrison, T. L., \& Einstein, G. O. (2010). Prospective memory: Are preparatory attentional processes necessary for a single focal cue? Memory \& Cognition, 38, 860-867. doi:10.3758/MC.38.7.860

Harrison, T. L., Mullet, H. G., Whiffen, K. N., Ousterhout, H., \& Einstein, G. O. (2013). Prospective memory: Effects of divided attention on spontaneous retrieval. Memory \& Cognition. doi:10.3758/s13421013-0357-y

Jacoby, L. L., \& Whitehouse, K. (1989). An illusion of memory: False recognition influenced by unconscious perception. Journal of Experimental Psychology: General, 118, 126-135. doi:10.1037/ 0096-3445.118.2.126

Johnston, W. A., Dark, V. J., \& Jacoby, L. L. (1985). Perceptual fluency recognition judgments. Journal of Experimental Psychology: Learning, Memory, and Cognition, 11, 3-11. doi:10.1037//02787393.11.1.3

Johnston, W. A., Hawley, K. J., \& Elliott, J. M. G. (1991). Contribution of perceptual fluency to recognition judgments. Journal of Experimental Psychology: Learning, Memory, and Cognition, 17, 210-223. doi:10.1037/0278-7393.17.2.210

Joordens, S., \& Merikle, P. M. (1992). False recognition and perception without awareness. Memory \& Cognition, 20, 151-159. doi:10. 3758/Bf03197164

Klinger, M. R. (2001). The roles of attention and awareness in the false recognition effect. American Journal of Psychology, 114, 93-114. doi: $10.2307 / 1423382$

Kurilla, B. P., \& Westerman, D. L. (2008). Processing fluency affects subjective claims of recollection. Memory \& Cognition, 36, 8292. doi:10.3758/Mc.36.1.82

Lee, J. H., \& McDaniel, M. A. (2013). Discrepancy-plus-search processes in prospective memory retrieval. Memory \& Cognition, 41, 443451. doi:10.3758/s13421-012-0273-6

Lloyd, M. E., Westerman, D. L., \& Miller, J. K. (2003). The fluency heuristic in recognition memory: The effect of repetition. Journal of Memory and Language, 48, 603-614. doi:10.1016/S0749596X(02)00535-1

Marsh, R. L., Hancock, T. W., \& Hicks, J. L. (2002). The demands of an ongoing activity influence the success of event-based prospective memory. Psychonomic Bulletin \& Review, 9, 604-610. doi:10.3758/ BF03196319

Marsh, R. L., Hicks, J. L., Cook, G. I., Hansen, J. S., \& Pallos, A. L. (2003). Interference to ongoing activities covaries with the characteristics of an event-based intention. Journal of Experimental Psychology: Learning, Memory, and Cognition, 29, 861-870. doi: 10.1037/0278-7393.29.5.861

McDaniel, M. A., \& Einstein, G. O. (2000). Strategic and automatic processes in prospective memory retrieval: A multiprocess framework. Applied Cognitive Psychology, 14, 127-144. doi:10.1002/ acp. 775

McDaniel, M. A., \& Einstein, G. O. (2007). Prospective memory: An overview and synthesis of an emerging field. Los Angeles: Sage.

McDaniel, M. A., Guynn, M. J., Einstein, G. O., \& Breneiser, J. (2004). Cue-Focused and reflexive-associative processes in prospective memory retrieval. Journal of Experimental Psychology: Learning, Memory, and Cognition, 30, 605-614. doi:10.1037/0278-7393.30. 3.605

Meier, B., Morger, V., \& Graf, P. (2003). Competition between automatic and controlled processes. Consciousness \& Cognition, 12, 309-319. doi:10.1016/S1053-8100(02)00069-7

Meier, B., \& Rey-Mermet, A. (2012). Beyond monitoring: After-effects of responding to prospective memory targets. Consciousness \& Cognition, 21, 1644-1653. doi:10.1016/j.concog.2012.09.003 
Meiser, T., \& Rummel, J. (2012). False prospective memory responses as indications of automatic processes in the initiation of delayed intentions. Consciousness \& Cognition, 21, 1509-1516. doi:10.1016/j. concog.2012.05.006

Meiser, T., \& Schult, J. C. (2008). On the automatic nature of the taskappropriate processing effect in event-based prospective memory. European Journal of Cognitive Psychology, 20, 290-311. doi:10. 1080/09541440701319068

Moors, A., \& De Houwer, J. (2006a). Automaticity: A theoretical and conceptual analysis. Psychological Bulletin, 132, 297-326. doi:10. 1037/0033-2909.132.2.297

Moors, A., \& De Houwer, J. (2006b). Problems with dividing the realm of processes. Psychological Inquiry, 17, 199-204.

Navon, D., \& Gopher, D. (1979). Economy of the human-processing system. Psychological Review, 86, 214-255. doi:10.1037/0033$295 \times .86 .3 .214$

Raven, J. C. (1962). Advanced progressive matrices: Set II. London: Lewis.

Redick, T. S., \& Lindsey, D. R. B. (2013). Complex span and n-back measures of working memory: A meta-analysis. Psychonomic Bulletin \& Review, 20, 1102-1113. doi:10.3758/s13423-013-0453-9

Rummel, J., Boywitt, C. D., \& Meiser, T. (2011). Assessing the validity of multinomial models using extraneous variables: An application to prospective memory. The Quarterly Journal of Experimental Psychology, 64, 2194-2210. doi:10.1080/17470218.2011.586708

Rummel, J., Einstein, G. O., \& Rampey, H. (2012). Implementation intention encoding in a prospective memory task enhances spontaneous retrieval of intentions. Memory, 20, 803-817. doi:10.1080/ 09658211.2012 .707214
Rummel, J., \& Meiser, T. (2013). The role of metacognition in prospective memory: Anticipated task demands influence attention allocation strategies. Consciousness \& Cognition, 22, 931-943. doi:10. 1016/j.concog.2013.06.006

Scullin, M. K., Bugg, J. M., \& McDaniel, M. A. (2012). Whoops, I did it again: Commission errors in prospective memory. Psychology \& Aging, 27, 46-53. doi:10.1037/a0026112

Smith, R. E. (2003). The cost of remembering to remember in eventbased prospective memory: Investigating the capacity demands of delayed intention performance. Journal of Experimental Psychology: Learning, Memory, and Cognition, 29, 347-361. doi: 10.1037/0278-7393.29.3.347

Smith, R. E., \& Bayen, U. J. (2004). A multinomial model of event-based prospective memory. Journal of Experimental Psychology: Learning, Memory, and Cognition, 30, 756-777. doi:10.1037/ 0278-7393.30.4.756

Wesslein, A. K., Rummel, J., \& Boywitt, C. D. (2014). Differential effects of cue-specificity and list-length on the prospective and retrospective prospective-memory components. Journal of Cognitive Psychology, 26, 135-146. doi:10.1080/20445911.2013.865628

Whittlesea, B. W. A., \& Williams, L. D. (2001a). The discrepancyattribution hypothesis: I. The heuristic basis of feelings and familiarity. Journal of Experimental Psychology: Learning, Memory, and Cognition, 27, 3-13. doi:10.1037/0278-7393.27.1.3

Whittlesea, B. W. A., \& Williams, L. D. (2001b). The discrepancyattribution hypothesis: II. Expectation, uncertainty, surprise, and feelings of familiarity. Journal of Experimental Psychology: Learning, Memory, and Cognition, 27, 14-33. doi:10.1037/02787393.27.1.14 